\title{
Dynamics of Reaction of Monoenergetic Atoms in a Thermal Gas*
}

\author{
By Aron Kuppermann, John Stevenson and Patricia O'Keefe
}

\author{
Gates and Crellin Laboratories of Chemistryt
}

California Institute of Technology, Pasadena, California 91109

Received 17th July, 1967

\begin{abstract}
When monoenergetic atoms are continuously introduced into a thermal gas, they can undergo deactivating, activating, and reactive collisions. The net result of such collisions is to establish a steady-state distribution of laboratory energies which, although not as sharp as the initial distribution, preserves some of its features, such as being centred at about the initial energy. The reactive collisions which occur under these conditions are characterized by the associated relative energy distribution function and the energy-dependent reaction cross section. As a result, as the initial laboratory energy of the atoms is experimentally varied, the relative energy distribution function can be made to sample appropriately the reaction cross section curve. Therefore, from measurements of the competition between reaction and thermalization processes as a function of initial atom laboratory energies, and from a knowledge of the non-reactive differential scattering cross section, it is possible to obtain information about the dependence on relative energy of the rotationally averaged reaction cross section. The appropriate Boltzmann steady-state equation needed to obtain this information is derived in this paper and solved for an assumed set of reactive and non-reactive cross sections. Distribution functions of relative energies are thereby obtained and used to indicate the usefulness of the suggested measurements.
\end{abstract}

\section{STEADY-STATE BOLTZMANN EQUATIONS}

We consider atoms $\mathrm{A}$ of mass $m_{1}$ continuously introduced into a thermal gas of molecules $\mathrm{B}$ of mass $m_{2}$ which is at temperature $T$. Let $R_{0}$ be the total rate of generation of atoms $\mathrm{A}$ and let $\varphi_{0}\left(\mathbf{v}_{1}\right)$ be the normalized distribution describing the laboratory velocity vectors $\mathbf{v}_{1}$ with which $A$ is introduced into the gas; $\phi_{0}\left(\mathbf{v}_{1}\right)$ will be considered spherically symmetrical but otherwise arbitrary. For the case of interest in the present paper it will be a rather narrow distribution function centred around an initial speed $v_{1}^{(0)}$. In particular it can be a $\delta$ function at $v_{1}^{(0)}$. The rate of introduction $R_{i}\left(\mathbf{v}_{1}\right) \mathrm{d} \mathbf{v}_{1}$ of atoms $\mathrm{A}$ in velocity range $\mathrm{v}_{1}$ to $\mathbf{v}_{1}+\mathrm{d} \mathbf{v}_{1}$ is therefore $R_{0} \phi_{0}\left(\mathrm{v}_{1}\right) \mathrm{d} \mathbf{v}_{1}$. Under steady-state conditions, let $g\left(\mathbf{v}_{1}\right) \mathrm{d} \mathbf{v}_{1}$ be the concentration of $\mathrm{D}$ atoms in that same velocity range. The rate $R^{(-)}\left(\mathbf{v}_{1}\right) \mathrm{d} \mathbf{v}_{1}$ at which atoms are removed from that range due to reactive and non-reactive collisions is given by

where

$$
R^{(-)}\left(\mathbf{v}_{1}\right) \mathrm{d} \mathbf{v}_{1}=k\left(\mathbf{v}_{1}\right) g\left(\mathbf{v}_{1}\right) \mathrm{d} \mathbf{v}_{1},
$$

$$
\begin{aligned}
k\left(\mathbf{v}_{1}\right) & =[\mathrm{B}] \int_{\mathbf{v}_{2}} \varphi_{T}\left(\mathbf{v}_{2}\right) v S_{t}(v) \mathrm{d} \mathbf{v}_{2}, \\
\varphi_{T}\left(\mathbf{v}_{2}\right) & =\left(\frac{m_{2}}{2 \pi k T}\right)^{\frac{3}{2}} \exp \left(-\frac{m_{2} v_{2}^{2}}{2 k T}\right), \\
\mathbf{v} & =\mathbf{v}_{1}-\mathbf{v}_{2}, \\
S_{t}(v) & =S_{n r}(v)+S_{r}(v) .
\end{aligned}
$$

* work supported in part by the U.S. Atomic Energy Commission. Report Code : CALT-532-13. $\dagger$ contribution no 3537 . 
In the expressions above, [B] is the number density of molecules $B, v_{2}$ is their laboratory velocity, $v$ is the relative velocity of $A$ with respect to $B, \varphi_{T}\left(v_{2}\right)$ is the normalized Maxwell distribution function of velocities $\mathbf{v}_{2}$ at temperature $T$, and $S_{t}(v), S_{n r}(v)$, and $S_{r}(v)$ are, respectively, the total, non-reactive and reactive cross sections of $\mathrm{A}$ and $\mathrm{B}$ at relative speed $v$. It is assumed that these cross sections have been averaged over the rotational motion of $\mathrm{B}$ at temperature $T$ and that otherwise only one internal quantum state of $\mathrm{A}$ and $\mathrm{B}$ are involved in the collisions. This condition is satisfied by the $\mathrm{D}+\mathrm{H}_{2}$ system considered below. The present formalism can easily be generalized so as to eliminate these restrictions.

The rate $R^{(+)}\left(\mathbf{v}_{1}\right) \mathrm{d} \mathbf{v}_{1}$ at which $\mathrm{D}$ atoms are inroduced into the range $\mathbf{v}_{1}$ to $\mathbf{v}_{1}+\mathrm{d} \mathbf{v}_{1}$ due to non-reactive collisions of $\mathrm{D}$ atoms at other velocities with $\mathrm{B}$ molecules is given by ${ }^{1}$

$$
R^{(+)}\left(\mathbf{v}_{1}\right) \mathrm{d} \mathbf{v}_{1}=[\mathrm{B}] \int_{\Sigma} g\left(\mathbf{v}_{1}^{\prime}\right) \varphi_{T}\left(\mathbf{v}_{2}^{\prime}\right) v^{\prime} \sigma_{n r}\left(v^{\prime}, \chi, \eta\right) \mathrm{d} \Omega \mathrm{d} \mathbf{v}_{1}^{\prime} \mathrm{d} \mathbf{v}_{2}^{\prime}
$$

where $\mathbf{v}_{1}^{\prime}$ and $\mathbf{v}_{2}^{\prime}$ are, respectively, the velocities of $A$ and $B$ before a non-reactive collision which results in velocities $\mathbf{v}_{1}$ and $\mathbf{v}_{2}$ after collision, $v^{\prime}$ is their relative speed before collision and $\Omega \equiv(\chi, \eta)$ is the direction of the relative velocity vector $\mathbf{v}$ after collision with respect to $\mathbf{v}^{\prime}$ and an arbitrarily chosen plane containing $\mathbf{v}^{\prime}$. Therefore $\chi$ is the scattering angle and $\eta$ the angle between the $\mathbf{v}^{\prime}, \mathbf{v}$ plane and the reference plane. There are a total of four velocity vectors $\mathbf{v}_{1}^{\prime}, \mathbf{v}_{2}^{\prime}, \mathbf{v}_{1}, \mathbf{v}_{2}$ or twelve scalar components which completely define the velocities of both particles before and after collision. Of these, however, only eight scalars are independent since conservation of centre of mass momentum as well as total energy establishes four relations among them. In eqn. $(1 \cdot 6)$ these eight scalars were taken as $\mathbf{v}_{1}^{\prime}, \mathbf{v}_{2}^{\prime}, \Omega(\chi, \eta)$, and $\Sigma$ is a surface in this eight-dimensional velocity space defined by the requirement that over it $\mathbf{v}_{1}$ be a constant. The integral in this expression is therefore five-dimensional and includes the contributions of all collisions between $A$ and $B$ which form $A$ in velocity range $v_{1}$ to $\mathbf{v}_{1}+\mathrm{d} \mathbf{v}_{1}$ after collision. These include both activating $\left(v_{1}>v_{1}^{\prime}\right)$ and thermalizing $\left(v_{1}<v_{1}^{\prime}\right)$ collisions, although for $v_{1}$ greater than the root mean square speed at temperature $T$, the effect of thermalizing collisions automatically predominates.

Under steady-state conditions, the net rate of introduction of $D$ atoms into range $\mathbf{v}_{1}$ to $\mathbf{v}_{1}+d \mathbf{v}_{1}$ due to all the processes described above is zero, by definition. The steady-state Boltzmann equation in laboratory velocity space is therefore

$$
\begin{aligned}
R_{i}\left(\mathbf{v}_{1}\right)-R^{(-)}\left(\mathbf{v}_{1}\right)+R^{(+)}\left(\mathbf{v}_{1}\right) & =0, \\
\text { or } \quad R_{0} \varphi_{0}\left(\mathbf{v}_{1}\right)-k\left(\mathbf{v}_{1}\right) g\left(\mathbf{v}_{1}\right)+R^{(+)}\left(\mathbf{v}_{1}\right) & =0 .
\end{aligned}
$$

We can transform this equation into laboratory speed space by multiplying it by $v_{1}^{2} \sin \theta_{1} \mathrm{~d} \theta_{1} \mathrm{~d} \varphi_{1}$ and integrating it over the spherical polar angles $\theta_{1}, \varphi_{1}$ of $\mathbf{v}_{1}$ with respect to an arbitrarily chosen laboratory system of reference. Making use of the spherically symmetric nature of the functions $\phi_{0}, k$ and $g$ we get :

where

$$
\begin{gathered}
R_{0} \varphi_{0}^{\prime}\left(v_{1}\right)-k^{\prime}\left(v_{1}\right) g^{\prime}\left(v_{1}\right)+R^{(+)}\left(v_{1}\right)=0, \\
\varphi_{0}^{\prime}\left(v_{1}\right)=4 \pi v_{1}^{2} \varphi_{0}\left(\mathbf{v}_{1}\right), \\
g^{\prime}\left(v_{1}\right)=4 \pi v_{1}^{2} g\left(\mathbf{v}_{1}\right), \\
k^{\prime}\left(v_{1}\right)=k\left(\mathbf{v}_{1}\right) .
\end{gathered}
$$

An explicit expression for $R^{(+)}\left(v_{1}\right)$ can now be obtained by calculating its integral over $v_{1}$ :

$$
\begin{aligned}
\int_{v_{1}} R^{\prime(+)}\left(v_{1}\right) \mathrm{d} v_{1}=\int_{\mathbf{v}_{1}} R^{(+)}\left(\mathbf{v}_{1}\right) \mathrm{d} \mathbf{v}_{1}= & \\
& {[\mathrm{B}] \int_{\mathbf{v}_{1}^{\prime}, \mathbf{v}_{2}^{\prime}, \Omega} g\left(\mathbf{v}_{1}^{\prime}\right) \varphi_{T}\left(\mathbf{v}_{2}^{\prime}\right) v^{\prime} \sigma_{n r}\left(v^{\prime}, \chi, \eta\right) \mathrm{d} \mathbf{v}_{1}^{\prime} \mathrm{d} \mathbf{v}_{2}^{\prime} \mathrm{d} \Omega . }
\end{aligned}
$$


The integral on the right-hand side of this expression now extends over the entire eight-dimensional velocity space $\mathbf{v}_{1}^{\prime}, \mathbf{v}_{2}^{\prime}, \Omega$ with no restriction on $\mathbf{v}_{1}$. We change integration variables from these to $\mathbf{v}_{1}^{\prime}, v_{2}^{\prime}, \varphi_{2}^{\prime}, v_{1}, \Omega$. This is equivalent to changing from variable $\theta_{2}^{\prime}$ to variable $v_{1}$, the other seven scalar variables $\mathbf{v}_{1}^{\prime}, v_{2}^{\prime}, \varphi_{2}^{\prime}, \Omega$ remaining unchanged. Here $\left(\theta_{2}^{\prime}, \varphi_{2}^{\prime}\right) \equiv \Omega_{2}^{\prime}$ represent the direction angles of $\mathbf{v}_{2}^{\prime}$. Let $J$ be the Jacobian of this transformation, for which

$$
\sin \theta_{2}^{\prime} \mathrm{d} \theta_{2}^{\prime}=|J| v_{1}^{2} \mathrm{~d} v_{1} \text {. }
$$

There results from this transformation

$$
R^{\prime(+)}\left(v_{1}\right)=[\mathrm{B}] \int_{\mathbf{v}_{1}^{\prime}, v_{2}^{\prime}, \varphi_{2}^{\prime}, \Omega} g\left(\mathbf{v}_{1}^{\prime}\right) \varphi_{T}\left(\mathbf{v}_{2}^{\prime}\right) v^{\prime} \sigma_{n r}\left(v^{\prime}, \chi, \eta\right)|J| v_{1}^{2} v_{2}^{\prime 2} \mathrm{~d} \mathbf{v}_{1}^{\prime} \mathrm{d} v_{2}^{\prime} \mathrm{d} \varphi_{2}^{\prime} \mathrm{d} \Omega .
$$

The integrand of eqn. (1.15) is considered to be a function of the eight independent scalars $\mathbf{v}_{1}^{\prime}, v_{2}^{\prime}, \varphi_{2}^{\prime}, \Omega$ and $v_{1}$. The integral is performed over the first seven of these variables, the last one remaining constant. Using eqn. (1.15) and (1.11) together with eqn. (1.9) gives :

$$
R_{0} \varphi_{0}^{\prime}\left(v_{1}\right)-k^{\prime}\left(v_{1}\right) g^{\prime}\left(v_{1}\right)+\int_{v_{1}^{\prime}} g^{\prime}\left(v_{1}^{\prime}\right) h^{\prime}\left(v_{1}^{\prime}, v_{1}\right) \mathrm{d} v_{1}^{\prime}=0
$$

where

$$
\begin{aligned}
& h^{\prime}\left(v_{1}^{\prime}, v_{1}\right)=\frac{[\mathrm{B}]}{2} \int_{v^{\prime}, \Omega} \varphi_{T}^{\prime}\left(v_{2}^{\prime}\right) v^{\prime} \sigma_{n r}\left(v^{\prime}, \chi, \eta\right)\left|\frac{\partial \cos \gamma^{\prime}}{\partial v_{1}}\right| \mathrm{d} v_{2}^{\prime} \mathrm{d} \Omega \\
& \varphi_{T}^{\prime}\left(v_{2}^{\prime}\right)=4 \pi v_{2}^{\prime 2} \varphi_{T}\left(\mathbf{v}_{2}^{\prime}\right) .
\end{aligned}
$$

To obtain eqn. (1.17), we chose as the axis for measuring $\theta_{2}^{\prime}, \varphi_{2}^{\prime}$ the direction of $\mathbf{v}_{1}^{\prime}$. Therefore, $\theta_{2}^{\prime}$ became the angle between $\mathbf{v}_{2}^{\prime}$ and $\mathbf{v}_{1}^{\prime}$, which we relabelled $\gamma^{\prime}$. The integrations over $\varphi_{2}^{\prime}, \theta_{1}^{\prime}$, and $\varphi_{1}^{\prime}$ were performed explicitly and furnished factors of $2 \pi$, 2 , and $2 \pi$, respectively, which when combined with the $4 \pi$ factors coming from the change from $g$ to $g^{\prime}$ and $\phi_{T}$ to $\phi_{T}^{\prime}$ gave the net factor of $1 / 2$ in the right-hand side of eqn. (1.17). The independent variables which appear in its integrand are thereby reduced to five, $v_{1}^{\prime}, v_{1}, v_{2}^{\prime}, \chi, \eta$. The partial derivative of $\gamma^{\prime}$ with respect to $v_{1}$ assumes therefore that $v_{1}^{\prime}, v_{2}^{\prime}, \chi$, and $\eta$ are kept constant.

We finally transform eqn. (1.16) into energy space by changing from variables $v_{1}$ and $v_{1}^{\prime}$ to

$$
\begin{aligned}
& E_{1}=\frac{1}{2} m_{1} v_{1}^{2}, \\
& E_{1}^{\prime}=\frac{1}{2} m_{1} v_{1}^{\prime 2} .
\end{aligned}
$$

There results the steady-state Boltzmann equation in energy space

where

$$
R_{0} \phi_{0}\left(E_{1}\right)-K\left(E_{1}\right) G\left(E_{1}\right)+\int_{E_{1}^{\prime}} G\left(E_{1}^{\prime}\right) H\left(E_{1}^{\prime}, E_{1}\right) \mathrm{d} E_{1}^{\prime}=0,
$$

$$
\begin{aligned}
& \phi_{0}\left(E_{1}\right)=\varphi_{0}^{\prime}\left(v_{1}\right) \mathrm{d} v_{1} / \mathrm{d} E_{1}, \\
& K\left(E_{1}\right)=k^{\prime}\left(v_{1}\right), \\
& G\left(E_{1}\right)=g^{\prime}\left(v_{1}\right) \mathrm{d} v_{1} / \mathrm{d} E_{1}, \\
& H\left(E_{1}^{\prime}, E_{1}\right)=h^{\prime}\left(v_{1}^{\prime}, v_{1}\right) \mathrm{d} v_{1} / \mathrm{d} E_{1} .
\end{aligned}
$$

In eqn. (1.22) through (1.25) the quantities $v_{1}$ and $v_{1}^{\prime}$ are considered functions of $E_{1}$ and $E_{1}^{\prime}$, respectively, obtained from eqn. (1.19) and (1.20). The distribution 
functions $\phi_{0}$ and $\varphi_{T}^{\prime}$ satisfy the normalization conditions,

$$
\begin{gathered}
\int_{E_{1}} \phi_{0}\left(E_{1}\right) \mathrm{d} E_{1}=1, \\
\int_{v_{2}} \varphi_{T}^{\prime}\left(v_{2}\right) \mathrm{d} v_{2}=1
\end{gathered}
$$

The distribution function $G\left(E_{1}\right)$ as defined above is non-normalized. $G\left(E_{1}\right) \mathrm{d} E_{1}$ represents the steady-state concentration of $\mathrm{A}$ atoms in laboratory energy range $E_{1}$ to $E_{1}+\mathrm{d} E_{1}$. Once $G\left(E_{1}\right)$ is obtained from a solution of eqn. (1.21), we can also obtain the normalized steady-state distribution function $F\left(E_{1}\right)$ of the A atom laboratory energy:

$$
F\left(E_{1}\right)=G\left(E_{1}\right) / \int_{E_{1}} G\left(E_{1}\right) \mathrm{d} E_{1} .
$$

It is possible to give a simple physical interpretation to eqn. (1.21). The first term times $\mathrm{d} E_{1}$ represents the initial rate of introduction of atoms $\mathrm{A}$ into laboratory energy range $E_{1}$ to $E_{1}+\mathrm{d} E_{1}$. The second term times $\mathrm{d} E_{1}$ represents the rate of removal of these atoms from that range due to all types of collisions with molecules $B$, reactive and non-reactive. Finally, the third term times $\mathrm{d} E_{1}$ represents the rate of formation of atoms $\mathrm{A}$ in that range due to all non-reactive collisions of such atoms whose laboratory energy before collision was $E_{1}^{\prime}$, integrated over all $E_{1}^{\prime}$ (larger than $E_{1}$ for thermalizing collisions and smaller than it for activating ones).

The following transformation permits one to obtain the distribution function in relative energies $f(E)$ from that in laboratory energies :

$$
f(E)=\int_{E_{1}} F\left(E_{1}\right) \phi_{\mathrm{BT}}\left(E_{1}, E\right) \mathrm{d} E_{1}
$$

where

$$
\phi_{\mathrm{BT}}\left(E_{1}, E\right)=\frac{1}{\mu v_{1}}\left(\frac{m_{2}}{2 \pi k T}\right)^{\frac{1}{2}}\left[\exp \left(-\frac{m_{2}\left(v-v_{1}\right)^{2}}{2 k T}\right)-\exp \left(-\frac{m_{2}\left(v+v_{1}\right)^{2}}{2 k T}\right)\right] .
$$

On the right-hand side of eqn. (1.30), $v_{1}$ and $v$ are considered to be functions of $E_{1}$ and $E$ according to eqn. (1.19) and (1.31), respectively.

$$
E=\frac{1}{2} \mu v^{2} \text {, }
$$

where $\mu$ is the reduced mass of the A, B particle pair. If all atoms A have the same energy $E$, i.e., are monoenergetic in the laboratory system, the distribution function of their relative energy with respect to thermal molecules $B$ is given by eqn. (1.30). This can be a very wide distribution, even if $k T$ is small compared to $E_{1}$. The reason is that if $\mathrm{A}$ and $\mathrm{B}$ have laboratory speeds $v_{1}$ and $v_{2}$, their relative speed can be anywhere between $v_{1}+v_{2}$ and $v_{1}-v_{2}$, depending on the directions of the corresponding velocity vectors. Therefore, their relative energies can be anywhere between $E_{\max }=\frac{1}{2} \mu\left(v_{1}+v_{2}\right)^{2}$ and $E_{\min }=\frac{1}{2} \mu\left(v_{1}-v_{2}\right)^{2}$ corresponding to a spread in relative energies $\Delta E=E_{\max }-E_{\min }=2 \mu v_{1} v_{2}$. Consequently, even though $v_{2}$ may be rather small, $\Delta E$ can be quite large if $v_{1}$ is large. If $v_{2}$ is substituted by $\left(3 k T / m_{2}\right)^{\frac{1}{2}}$, the root mean square speed of B at temperature $T$, the resulting $\Delta E$ is $2 \mu\left(6 k T E_{1} / m_{1} m_{2}\right)^{\frac{1}{2}}$. This is a measure of the width of the distribution function given by eqn. (1.30). For $E_{1} \gg k T$ this function has a peak at $E$ approximately equal to $E_{1}$, and its width is much larger than $k T$ if $m_{1} / m_{2}$ and $m_{2} / m_{1}$ are not much smaller than unity. For example, for the $\mathrm{D}+\mathrm{H}_{2}$ system, even at temperatures as low as $200^{\circ} \mathrm{K}$, this width is $0.45 \mathrm{eV}$ for $E_{1}=2 \mathrm{eV}$ (which corresponds to a most probable relative energy 
of $1 \mathrm{eV}$ ). In summary, a relatively small thermal spread in the B laboratory speed can produce a large spread in the relative energy of $A$ with respect to $B$, and if one assumes that the $B$ are stationary, very serious errors can result.

It is possible from eqn. (1.21) to obtain a rigorous steady-state Boltzmann equation directly in relative energies. Indeed, multiplication by $\varphi_{\mathrm{BT}}\left(E_{1}, E\right) / K\left(E_{1}\right)$ and integration over $E_{1}$ furnishes

$$
R_{0} \phi_{0}^{\prime}(E)-G^{\prime}(E)+\int_{E_{1}} \frac{\varphi_{\mathrm{BT}}\left(E_{1}, E\right)}{K\left(E_{1}\right)}\left[\int_{E_{1}^{\prime}} G\left(E_{1}^{\prime}\right) H\left(E_{1}^{\prime}, E_{1}\right) \mathrm{d} E_{1}^{\prime}\right] \mathrm{d} E_{1}=0
$$

where

$$
\phi_{0}^{\prime}(E)=\int_{E_{1}} \phi_{0}\left(E_{1}\right) \frac{\varphi_{\mathrm{BT}}\left(E_{1}, E\right)}{K\left(E_{1}\right)} d E_{1}
$$

and $G^{\prime}(E)$ is the non-normalized distribution function in relative energy. $G^{\prime}(E) \mathrm{d} E$ represents the steady-state concentration of atoms $\mathbf{A}$ whose energy relative to $\mathrm{B}$ is in the range $E$ to $E+\mathrm{d} E . G^{\prime}(E)$ is related to $G\left(E_{1}\right)$ by

$$
G^{\prime}(E)=\int_{E_{1}} G\left(E_{1}\right) \varphi_{\mathrm{BT}}\left(E_{1}, E\right) \mathrm{d} E_{1}
$$

The inverse transform is

$$
G\left(E_{1}\right)=\int_{E} G^{\prime}(E) \varphi_{\mathrm{BT}}^{\prime}\left(E, E_{1}\right) \mathrm{d} E
$$

where

$$
\varphi_{\mathrm{BT}}^{\prime}\left(E, E_{1}\right)=\frac{1}{m_{1} v}\left(\frac{m_{2}}{2 \pi k T}\right)^{\frac{1}{2}}\left[\exp \left(-\frac{m_{2}\left(v_{1}-v\right)^{2}}{2 k T}\right)-\exp \left(-\frac{m_{2}\left(v_{1}+v\right)^{2}}{2 k T}\right)\right]
$$

In the right-hand side of this last equation, $v_{1}$ and $v$ are the functions of $E_{1}$ and $E$ given by eqn. (1.19) and (1.31), respectively. The following normalization properties are satisfied by $\varphi_{\mathrm{Br}}$ and $\varphi_{\mathrm{BT}}^{\prime}$ :

$$
\int_{E} \varphi_{\mathrm{BT}}\left(E_{1}, E\right) \mathrm{d} E=\int \varphi_{\mathrm{BT}}^{\prime}\left(E, E_{1}\right) \mathrm{d} E_{1}=1
$$

Replacement of the dummy variable $E$ by $E^{\prime}$ in eqn. (1.35) followed by substitution into the last term of the right-hand side of eqn. (1.32) and a change in the order of the integrations furnishes finally

where

$$
R_{0} \phi_{0}^{\prime}(E)-G^{\prime}(E)+\int_{E^{\prime}} G^{\prime}\left(E^{\prime}\right) H^{\prime}\left(E^{\prime}, E\right) \mathrm{d} E^{\prime}=0
$$

$$
H^{\prime}\left(E^{\prime}, E\right)=\int_{E_{1}^{\prime}, E_{1}} H\left(E_{1}^{\prime}, E_{1}\right) \frac{\varphi_{\mathrm{BT}}\left(E_{1}, E\right) \varphi_{\mathrm{BT}}^{\prime}\left(E^{\prime}, E_{1}^{\prime}\right)}{K\left(E_{1}\right)} \mathrm{d} E_{1}^{\prime} \mathrm{d} E_{1}
$$

Eqn. (1.38) is the steady-state Boltzmann equation in relative energies sought. Since the functions $\phi_{0}^{\prime}(E)$ and $H^{\prime}\left(E^{\prime}, E\right)$ are known, it can in principle be solved to give $G^{\prime}(E)$ directly. In terms of the latter, the normalized steady-state distribution function $f(E)$ is given by

$$
f(E)=G^{\prime}(E) / \int_{E} G^{\prime}(E) \mathrm{d} E
$$


An implied assumption in the derivation of eqn. (1.21) was that the amount of energy introduced into the $A+B$ system via the kinetic energy of atoms $A$ was sufficiently small so as not to disturb the thermal distribution of energies of molecules B. It is always possible to satisfy this assumption by making [B] sufficiently large.

Eqn. (1.21) can be easily modified to include the effect of one or more additional reactants $C_{i}$. To this effect it suffices to add to the right-hand side of eqn. (1.2) and (1.17) similar terms for these substances. If only one additional reactant $C$ is considered, and if it is much more effective than $B$ in reacting with thermalized $A$ atoms, then a small $[C] /[B]$ ratio can suffice to scavenge such thermalized atoms without essentially contributing to the thermalization process. Under these conditions, the entire effect of introducing small amounts of $C$ into the $A+B$ system can be approximated quite well by adding to the right-hand side of eqn. (1.2) a similar term for $\mathrm{C}$ which, however, includes only the reactive part $S_{r}^{\prime}\left(v_{c}\right)$ of the $\mathrm{A}+\mathrm{C}$ cross section, and leaving all other terms unchanged. Here $v_{c}$ is the relative speed of $\mathrm{A}$ with respect to $\mathrm{C}$. The calculations described in Section 2 correspond to this case.

The generalization of the relative-energy Boltzmann eqn. (1.38) for a multireactant system is also easily obtained from the corresponding generalized form of eqn. (1.21).

\section{SOLUTION OF THE STEADY-STATE BOLTZMANN EQUATION IN LABORATORY ENERGY}

Given the reactive cross-sections $S_{r}(v)$ and $S_{r}^{\prime}\left(v_{c}\right)$ and the differential non-reactive cross section $\sigma_{n r}(v, \chi, \eta)$, as well as the initial rate $R_{0}$ of production of D atoms' initial distribution function $\phi_{0}\left(E_{1}\right)$, temperature $T$ and $\mathrm{B}$ and $\mathrm{C}$ molecule number densities [B] and [C], it is possible to solve the corresponding generalized form of the steadystate Boltzmann eqn. (1.21). We call $R_{0} \phi_{0}$ the source term, $K G$ the sink term and $\int G H \mathrm{~d} E_{1}^{\prime}$ the collision integral. This equation is a linear integral equation of the third kind, in which the known function $H\left(E_{1}^{\prime}, E_{1}\right)$ is called the kernel. It can be solved numerically by several methods, one of which is the Liouville-Neumann series. ${ }^{2}$ This consists essentially in writing the unknown function $G\left(E_{1}\right)$ as

$$
G\left(E_{1}\right)=\sum_{i=0}^{\infty} G_{i}\left(E_{1}\right)
$$

and determining the $G_{i}$ by the relationships,

$$
\left.\begin{array}{rl}
G_{0}\left(E_{1}\right) & =R_{0} \phi_{0}\left(E_{1}\right) / K\left(E_{1}\right) \\
G_{i}\left(E_{1}\right) & =I_{i-1}\left(E_{1}\right) / K\left(E_{1}\right) \quad i=1,2 \ldots \\
I_{i-1}\left(E_{1}\right) & =\int G_{i-1}\left(E_{1}^{\prime}\right) H\left(E_{1}^{\prime}, E_{1}\right) \mathrm{d} E_{1}^{\prime}
\end{array}\right\}
$$

We have obtained such solutions, but not for an exact kernel $H$ as defined by eqn. (1.25), (1.17), and (1.18). Instead, we used an approximate kernel, as described below. The reason for doing this is that the purpose of the calculations reported in this paper was not to obtain accurate values of $f(E)$ for any particular $\mathrm{A}+\mathrm{B}$ system, but to develop instead information about the general shape of $f(E)$ and its variation with $E^{(0)}$, the initial laboratory energy with which the $\mathrm{A}$ atoms are injected into the system. This purpose is adequately and more easily accomplished by picking a simplified kernel which preserves, nevertheless, the main properties of kernels which describe real systems. To do this we notice, from eqn. (1.17), that $\sigma_{n r}\left(v^{\prime}, \chi, \eta\right)$ and $\varphi_{T}^{\prime}\left(v_{2}^{\prime}\right)$ appear as part of the integrand. We know that $\sigma_{n r}$ is a rather sharp function of $\chi$, meaning 
that the non-reactive differential cross sections for small angular deflections (corresponding to large impact parameters) tend to be quite large and fall off very rapidly as the deflection angle increases. This means that the contributions to $h^{\prime}$ from relatively small energy losses tend to play a major role. This suggests that $H\left(E_{1}^{\prime}, E_{1}\right)$ be made a gaussian function centred at $E_{1}^{\prime}=E_{1}$. In addition, $\varphi_{T}^{\prime}\left(v_{2}^{\prime}\right)$ may produce in $E_{1}^{\prime}$ a spread of the order of

$$
\Delta E_{1}^{\prime}=\sqrt{ } 2 m_{1} k T / m_{2}
$$

We therefore chose $H\left(E_{1}^{\prime}, E_{1}\right)$ as having the form

$$
H\left(E_{1}^{\prime}, E_{1}\right)=A \varphi_{\mathrm{H}}\left(E_{1}^{\prime}, E_{1}\right)
$$

where

$$
\varphi_{\mathrm{H}}\left(E_{1}^{\prime}, E_{1}\right)=\frac{1}{(2 \pi)^{\frac{1}{2}} \Delta E_{1}^{\prime}} \exp \left(-\frac{\left(E_{1}^{\prime}-E_{1}\right)^{2}}{2\left(\Delta E_{1}^{\prime}\right)^{2}}\right)
$$

is a normalized gaussian function. In order to determine $A$, we used the following relationship between $K_{n r}$ and $H$ :

$$
K_{n r}\left(E_{1}\right)=\int_{E_{1}^{\prime}} H\left(E_{1}, E_{1}^{\prime}\right) \mathrm{d} E_{1}^{\prime} .
$$

In this expression, $K_{n r}$ refers to the part of $K$ obtained replacing $S_{t}(v)$ by $S_{n r}(v)$ in eqn. (1.2), (1.12), and (1.23). Eqn. (2.6) is simple to prove and states essentially, when both sides of it are multiplied by $G\left(E_{1}\right) \mathrm{d} E_{1}$, that the rate of removal of A atoms out of energy range $E_{1}$ to $E_{1}+\mathrm{d} E_{1}$ due to non-reactive collisions with molecules $\mathrm{B}$ is equal to the rate at which atoms $A$ are formed with energy $E_{1}^{\prime}$ due to such collisions, integrated over all possible values of that final energy. Substitution of eqn. (2.4) into eqn. (2.6) gives

$$
K_{n r}\left(E_{1}\right)=A .
$$

We conclude that for the kernel defined by eqn. (2.4), $K_{n r}$ must be a constant, independent of $E_{1}$. Returning to the definition of $K_{n r}\left(E_{1}\right)$ given above, this implies that

$$
v S_{n r}(v)=\alpha \text {, }
$$

where $\alpha$ is a constant independent of $v$. Indeed, substitution of eqn. (2.8) into the definition of $K_{n r}\left(E_{1}\right)$ gives

$$
A=\alpha[\mathrm{B}] \text {. }
$$

Therefore, eqn. (2.4) and (2.7) through (2.9) completely define the kernel and sink terms for which we did our calculations. The main advantage in introducing these simplifications when making initial calculations is to avoid the time-consuming threedimensional numerical integral indicated by eqn. (1.17) which would otherwise have to be performed. In trying to obtain quantitative reaction cross sections from experimental data the use of that equation is required.

In addition to the $H$ and $K_{n r}$ just mentioned, we chose the following form for the two cross sections describing the reaction of $\mathrm{A}$ with $\mathrm{B}$ and with the thermal atom scavenger $\mathrm{C}$ :

$$
S_{r}(v)= \begin{cases}\pi b^{2}\left(1-E_{0} / E\right) & \text { for } E \geqslant E_{0}, \\ 0 & \text { for } 0 \leqslant E \leqslant E_{0},\end{cases}
$$

where $E$ is related to $v$ by eqn. (1.31), and

$$
S_{r}^{\prime}\left(v_{c}\right)=\gamma / v_{c},
$$


where $\gamma$ is a constant independent of $v_{c}$. Eqn. (2.10) is the usual hard sphere line of centres reactive cross section with relative threshold energy $E_{0}$ and eqn. (2.11) represents a cross section which furnishes a thermal rate constant independent of temperature, as is essentially the case for many scavengers of thermalized atoms.

\section{RESULTS AND DISCUSSION}

Calculations of $f(E)$ were performed using the scheme described in $\$ 2$ and picking values of the several parameters of the correct order of magnitude to describe the system $\mathrm{D}+\mathrm{H}_{2}$ with $\mathrm{DI}$ as a scavenger. The parameter $\alpha$ was chosen so as to make $S_{n r}(v)=46.5 \AA^{2}$ for $v=6.2 \times 10^{5} \mathrm{~cm} / \mathrm{sec}$. $\left[\mathrm{H}_{2}\right]$ was made $3.2 \times 10^{18}$ molecules $/ \mathrm{cm}^{3}$ corresponding to a partial pressure of 100 torr at the chosen temperature of $300^{\circ} \mathrm{K}$. $\pi b^{2}$ was chosen as $1 \AA^{2}$ and $E_{0}$ was made equal to $0.3 \mathrm{eV}$. $\gamma$ was chosen so that at $v_{c}=1.56 \times 10^{5} \mathrm{~cm} / \mathrm{sec}, S_{r}^{\prime}\left(v_{c}\right)=0.1 \AA^{2}$. The value of $R_{0}$ was taken as $10^{10} \mathrm{D}$ atoms $/ \mathrm{cm}^{3} \mathrm{sec}$ corresponding to the experimental conditions for photolyzing mixtures of $\mathrm{DI}+\mathrm{H}_{2}$ used in a study of the $\mathrm{D}+\mathrm{H}_{2} \rightarrow \mathrm{DH}+\mathrm{H}$ reaction. ${ }^{3}$ The normalized source term $\phi_{0}\left(E_{1}\right)$ was chosen as a gaussian function centred at $E_{1}^{(0)}$ and with root mean square width of $0.03 \mathrm{eV}$, also corresponding to the experimental conditions just mentioned. ${ }^{3}$ The central $\mathrm{D}$ atom laboratory energy $E_{1}^{(0)}$ was given the values $0.4,0.7$ and $1.2 \mathrm{eV}$, corresponding to most probable values of the relative energy of $0.20,0.35$, and $0.60 \mathrm{eV}$, respectively. Finally, the $[\mathrm{DI}] /\left[\mathrm{H}_{2}\right]$ ratio was varied between $0 \cdot 1$ and $2 \cdot 5$. It was found that the resulting distribution functions were essentially independent of this ratio. This can be understood on the basis that the main constribution to $K$ comes from $S_{n r}(v)$, the contributions due to $S_{r}(v)$ and $S_{r}^{\prime}\left(v_{c}\right)$ being negligible in comparison. This means that the $f(E)$ should be essentially independent of the reaction cross sections, being determined mainly by the differential non-reactive cross section. This is an important point, since it enormously simplifies the procedure for extracting reaction cross sections from experimental data, as pointed out below.

In fig. 1 are depicted several of the curves obtained. Curve $S_{r}$ represents the reaction cross section defined by eqn. (2.10) with values of the parameters as chosen above. Curve $f_{T} / 5$ represents a normalized Maxwellian distribution of energies at $300^{\circ} \mathrm{K}$, with the ordinates divided by 5 . For $E \geqslant E_{0}=0.3 \mathrm{eV}$, the values of its ordinates are essentially zero compared to its maximum value. Curves $a, b$, and $c$ correspond to $E_{1}^{(0)}=0.4,0.7$, and $1.2 \mathrm{eV}$. They have maxima close to $E=0.2$, $0 \cdot 35$, and $0.6 \mathrm{eV}$ respectively, as expected, since the reduced mass of the $\mathrm{D}+\mathrm{H}_{2}$ system is $\frac{1}{2}$ of the mass of a D atom. The most important point is that these curves have large ordinates (in units of their maximum values) in regions where the cross section $S_{r}(v)$ is appreciable. Therefore, scanning $E_{1}^{(0)}$ is an excellent way of sampling this reaction cross section.

In fig. 2, curve $c$ is depicted again, together with curve $d$ which is the corresponding source distribution function $\phi_{0}\left(E_{1}\right)$ transformed into laboratory energies. One can see that the broadening in going from $d$ to $c$, produced by the multiple thermalizing collisions, is not large. This means that the results of experiments performed under these conditions should not be too unlike those obtained when a $\mathrm{D}$ atom beam collides with a thermal $\mathrm{H}_{2}$ gas target under conditions such that only a single collision occurs. In other words, the multiple collision nature of the photolysis experiments mentioned does not make them extremely different from single collision atomic beam experiments.

Finally, we analyze the consequences of the distribution functions $f(E)$ being determined almost exclusively by the differential and total non-reactive cross sections, and essentially independent of the reactive ones. This means that once the nonreactive cross sections are known, the $f(E)$ can be calculated by the methods described 
above. Then the reaction cross section $S_{r}(v)$ can be obtained simply by unfolding it with respect to $f(E)$ in such a way as to produce extents of hot reaction and thermalization which agree with experiment. In view of the nature of the dependence

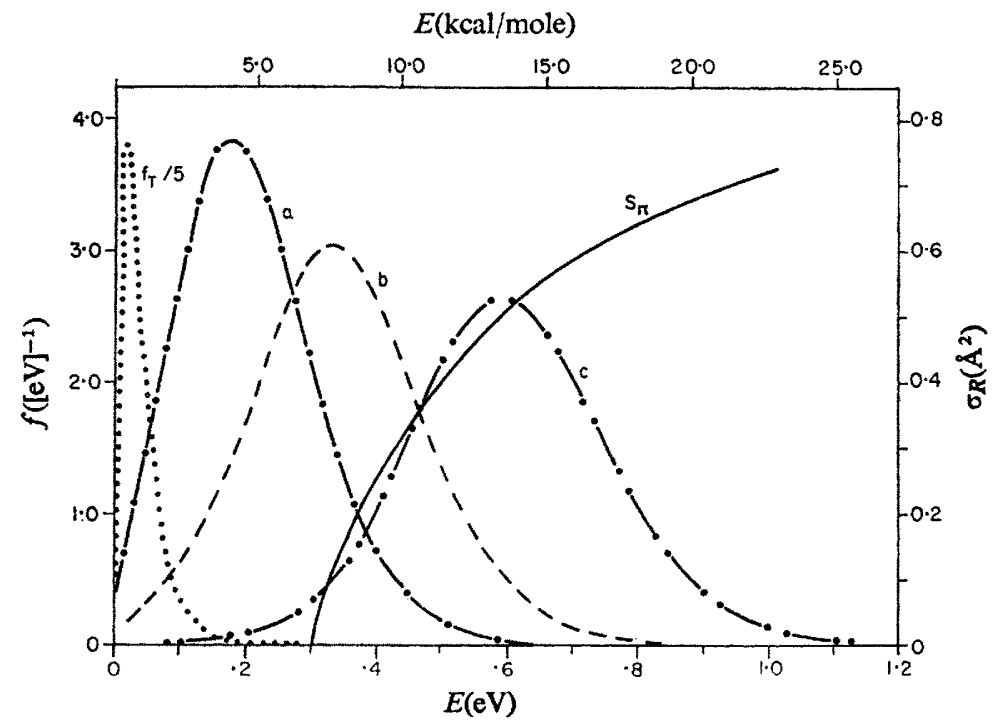

FIG. 1.-Variation of distribution functions and cross section with relative energy. $f_{T}$, normalized Maxwellian distribution at $300^{\circ} \mathrm{K}$; ordinates have been divided by $5 . \quad a, b$, and $c$, normalized $f(E)$ curves for $E_{1}^{(0)}=0.4,0.7,1.2 \mathrm{eV}$, respectively; see text for values of other parameters. $S_{r}$, assumed reactive cross section curve for $\mathrm{D}+\mathrm{H}_{2} \rightarrow \mathrm{DH}+\mathrm{H}$; see text for value of parameters.

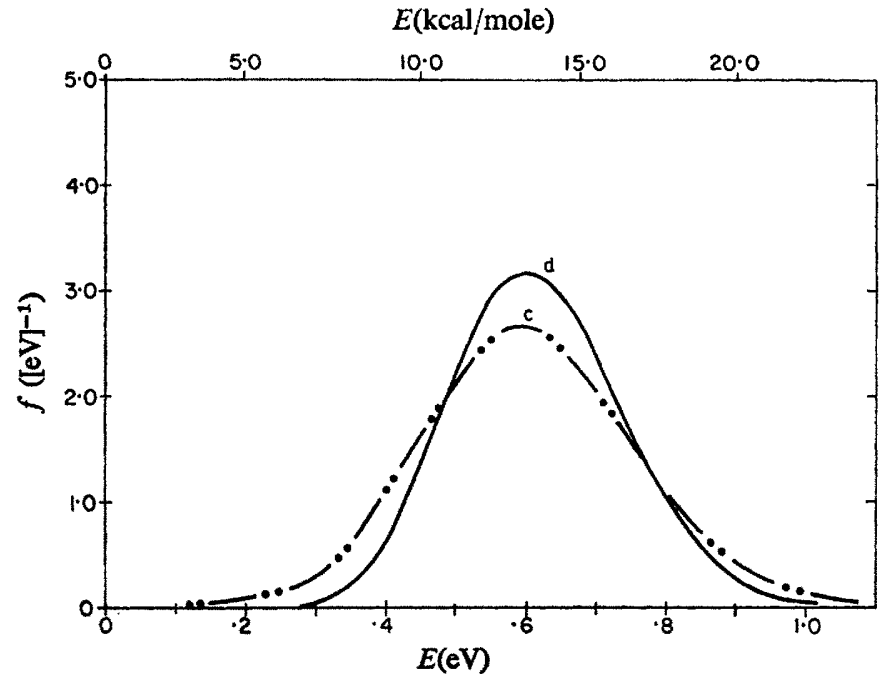

Fio. 2.-Initial and steady-state distribution functions of relative energies. $E_{1}^{(0)}=1.2 \mathrm{eV} ; d$, initial distribution function; $c$, steady-state distribution function.

of $f(E)$ on $E_{1}^{(0)}$ described above, such unfolding should be devoid of difficulties and produce accurate results. The procedures outlined above are presently being used to determine $S_{r}(v)$ for the $\mathrm{D}+\mathrm{H}_{2} \rightarrow \mathrm{DH}+\mathrm{H}$ reaction from experimental [DH]/[D $]$ 
ratios obtained in the photolyses of $\mathrm{DI}+\mathrm{H}_{2}$ mixtures with monochromatic light of variable wave-length.

One of the author (A. K.) thanks Prof. R. N. Porter for stimulating discussions on this problem. We also express our appreciation to Prof. John M. White and Mr. Joel Goldberg who participated in the early stages of this work.

1 J. O. Hirschfelder, C. F. Curtiss and R. B. Bird, Molecular Theory of Gases and Liquids, (John Wiley and Sons, Inc., New York, 1964), p. 502.

${ }^{2} \mathrm{H}$. Margenau and G. M. Murphy, The Mathematics of Physics and Chemistry, (D. Van Nostrand Company, Inc., New York, 1956), p. 521.

${ }^{3}$ A. Kuppermann and J. M. White, J. Chem. Physics, 1966, 44, 4352. 